\title{
RESOLVENT AND LATTICE POINTS ON SYMMETRIC SPACES OF STRICTLY NEGATIVE CURVATURE
}

\author{
R.W.BRUGGEMAN, R.J. MIATELLO, AND N.R. WALLACH
}

\begin{abstract}
We study the asymptotics of the lattice point counting function $N(x, y ; r)=\#\{\gamma \in \Gamma: d(x, \gamma y)\}$ for $X$ a Riemannian symmetric space of rank one and $\Gamma$ a discontinuous group of motions in $X$, such that $\Gamma \backslash X$ has finite volume. We show that

$N(x, y ; r)=\sum_{j=0}^{m} c_{j} \varphi_{j}(x) \varphi_{j}(y) e^{\left(\rho+\nu_{j}\right) r}+O_{x, y, \varepsilon}\left(e^{(2 \rho n /(n+1)+\varepsilon) r}\right)$

as $r \rightarrow \infty$, for each $\varepsilon>0$. The constant $2 \rho$ corresponds to the sum of the positive roots of the Lie group associated to $X$, and $n=\operatorname{dim} X$. The sum in the main term runs over a system of orthonormal eigenfunctions of the Laplacian $\varphi_{j} \in L^{2}(\Gamma \backslash X)$, corresponding to the exceptional eigenvalues.
\end{abstract}

\section{INTRODUCTION}

Let $X$ be a Riemannian symmetric space of strictly negative curvature. Then $X=G / K$, with $G$ a semisimple Lie group of rank one over $\mathrm{R}$ and $K$ a maximal compact subgroup of $G$. Let $\Gamma$ be a discrete subgroup of $G$ of finite covolume. A classical problem is the determination of the asymptotic behavior of the counting function

$$
N(r)=N(x, y ; r)=\operatorname{card}\{\gamma \in \Gamma: d(x, \gamma y)<r\},
$$

as $r \rightarrow \infty$, for fixed $x, y \in X$. This problem has been investigated by many authors. For instance, in the case when $\Gamma$ is cocompact, it was treated in $[\mathrm{Hbr}],[\mathrm{Ma}],[\mathrm{G}]$, and in $[\mathrm{B}]$. In the case when $\Gamma$ is of finite covolume, the main results in the literature concentrate on the case of hyperbolic $n$-space $H^{n}$. There are results of Selberg (unpublished) and Patterson for $X=H^{2}$, and of Lax-Phillips for $H^{n}$. (See [Pa], [LP]; see also [Le] and [MW], §5.) The main theme in these papers links the asymptotic behavior of $N(r)$ with spectral data of the Laplacian on $\Gamma \backslash X$. There is a main term $N(r) \sim c_{0} e^{2 \rho r}$, where $c_{0}, \rho$ are positive constants depending only on $X\left(2 \rho=n-1\right.$ if $\left.X=H^{n}\right)$. More precisely,

$$
N(r)=c_{0} e^{2 \rho r}+\sum_{j} c_{j} \varphi_{j}(x) \varphi_{j}(y) e^{\left(\rho+\nu_{j}\right) r}+E(r)
$$

1991 Mathematics Subject Classification. 11P21 (53C35, 58G25, 22E40).

Miatello: Partially supported by Conicet, Conicor, Secyt U.N.C., and Fundación Antorchas.

Wallach: Research partially supported by an NSF grant. 
where $0<\nu_{j}<\rho, 1 \quad j \quad m$ are chosen such that the $\lambda\left(\nu_{j}\right):=\rho^{2}-\nu_{j}^{2}$ are the eigenvalues of $-\Delta$ on $\Gamma \backslash X$ with value in $\left(0, \rho^{2}\right)$, with corresponding orthonormal eigenfunctions $\varphi_{j}$. These eigenvalues are called exceptional. Furthermore, the $c_{j}$ 's are constants involving values of Gamma functions on the $\nu_{j}$ 's, and the constant $b$ satisfies $0<b<\rho$. Finally $E(r)=O\left(r^{a} e^{(b+\rho) r}\right)$, for some positive constants $a, b$.

In the case of $H^{n}$ for $n>2$, the best result is due to Lax-Phillips ([LP]), who have proved that $E(r)=O\left(r^{3 /(n+1)} e^{\left(b_{0}+\rho\right) r}\right)$ with $b_{0}=\frac{(n-1)^{2}}{2(n+1)}$. (Actually they prove this result under a condition weaker than finite volume.)

In the present paper we will use some results in [MW] on the meromorphic continuation of the resolvent of the Laplacian on $X$ and $\Gamma \backslash X$, to obtain an estimate for $N(r)$, for any $X$ and $\Gamma$ as above, with $c_{0}=\frac{\zeta 2^{1-n}}{2 \rho \operatorname{vol}(\Gamma \backslash X)}$, $\rho=\rho(G)$ and $c_{j}=\frac{c\left(\nu_{j}\right) \zeta 2^{1-n}}{\nu_{j}+\rho}$. Here $\zeta$ is a constant depending only on $G$ and $c(\nu)$ denotes the Harish Chandra $c$-function. Theorem 4.1 gives the full statement; see Remark 4.2, (i) for a comparison with [LP]. In the case of $H^{n}$, we obtain $E(r)=e^{(b+\rho) r}$ for each $b>b_{0}=\frac{(n-1)^{2}}{2(n+1)}$. Our methods are different from those in $[\mathrm{LP}]$, who use the wave equation. We shall follow the approach in $[\mathrm{MW}]$, via functions related to the resolvent of $\Delta$. The main tools in the proof are certain estimates of a truncation of the resolvent, Hörmander's theorem on the spectral function of an elliptic operator, and a suitable Tauberian theorem (Proposition 5.1).

\section{Preliminaries}

Let $G$ be a connected semisimple Lie group of real rank one and finite center. Let $K$ be a maximal compact subgroup of $G$ with corresponding Cartan involution $\theta$ and Cartan decomposition $\mathrm{g}=\mathrm{k} \oplus \mathrm{p}$. Fix $G=N A K$ an Iwasawa decomposition of $G$ and let $\mathrm{g}=\mathrm{k} \oplus \mathrm{a} \oplus \mathrm{n}$ be the corresponding decomposition at the Lie algebra level. Let $M$ be the centralizer of $A$ in $K$, $P=M A N$ and denote by $\mathrm{m}$ the Lie algebra of $M$. (Note that $\mathrm{p}$ is not the Lie algebra of $P$.) If $\alpha$ is the simple root of $(P, A)$ then $\mathrm{n}=\mathrm{n}_{\alpha} \oplus \mathrm{n}_{2 \alpha}$ where $\mathrm{n}_{\alpha}\left(\right.$ resp. $\left.\mathrm{n}_{2 \alpha}\right)$ is the root space associated to $\alpha($ resp. $2 \alpha)$. Set $p=\operatorname{dim}\left(\mathrm{n}_{\alpha}\right)$, $q=\operatorname{dim}\left(\mathrm{n}_{2 \alpha}\right), \rho=\frac{1}{2}(p+2 q) \alpha$. Then $n=p+q+1=\operatorname{dim} X, X=G / K$.

Fix $H_{0} \in$ a such that $\alpha\left(H_{0}\right)=1$. We shall work with the multiple $B$ of the Killing form of $\mathrm{g}, B_{\mathrm{K}}$, for which $B\left(H_{0}, H_{0}\right)=1$. Note that $B_{\mathrm{K}}\left(H_{0}, H_{0}\right)=$ $2 p+8 q$ and hence $B=\frac{1}{2 p+8 q} B_{\mathrm{K}}$.

With this choice, $H_{\alpha}=H_{0}$. If $X, Y \in \mathrm{g}$, let $\langle X, Y\rangle=-B(X, \theta Y)$. Then $\langle$,$\rangle defines an inner product on \mathrm{g}$ which coincides with $B$ on $\mathrm{a}$. We will also denote by $\langle$,$\rangle the bilinear extension of B$ to $a_{c}=a \nwarrow_{R} C$, and its dual form on $\mathrm{a}_{c}^{*}$. All characters of $A$ are of the form $a \mapsto a^{\nu \alpha}$ with $\nu \in \mathrm{C}$. Often we write $a^{\nu}$ instead of $a^{\nu \alpha}$, that is, we identify $\mathrm{a}_{c}$ and $\mathrm{a}_{c}^{*}$ with $\mathrm{C}$ via the maps $H \rightarrow B\left(H, H_{0}\right), \lambda \rightarrow \lambda\left(H_{0}\right)$. We also set, if $t \in \mathbf{R}, a_{t}=\exp \left(t H_{0}\right)$, and $a^{\alpha}=e^{\alpha(\log a)}$. Thus $a_{t}^{\alpha}=e^{t}$. If $A^{+}=\left\{a_{t}: t>0\right\}$, we have the decomposition $G=G^{+} \cup K$, a disjoint union, with $G^{+}=K A^{+} K$. If 
$g \in G^{+}$, and $g=k_{1}(g) a(g) k_{2}(g)$, with $k_{j}(g) \in K, j=1,2, a(g) \in A^{+}$, then $a(g)$ is uniquely determined by $g$.

We choose the Riemannian metric $d x$ on $X=G / K$, induced by the restriction $\left.B\right|_{\mathrm{p}}$ of $B$ to $\mathrm{p}$. We then have that $d(x K, y K)=B\left(t H_{0}, t H_{0}\right)^{1 / 2}=$ $t$ if $x, y \in G$ and $a\left(x^{-1} y\right)=\exp \left(t H_{0}\right)$, with $t=t(x, y)>0$. Given our choices this implies that

$$
a\left(x^{-1} y\right)^{\alpha}=e^{d(x K, y K)}=e^{t}
$$

In the sequel, we will often use elements of $G$ to denote the corresponding elements of $X=G / K$.

We let $d g$ (resp. $d \bar{g}$ ) be the Haar measure on $G$ (resp. $G / K)$ such that $d g=\gamma\left(a_{t}\right) d k_{1} d a d k_{2}$ on $K A^{+} K$, where $\gamma(a)=\left(a^{\alpha}-a^{-\alpha}\right)^{p}\left(a^{2 \alpha}-a^{-2 \alpha}\right)^{q}$, and $d k$ is the Haar measure on $K$ normalized so that $K$ has volume 1 .

We claim that $d x=\zeta 2^{-p-q} d \bar{g}$, where $\zeta=\operatorname{vol}(K / M)$, the volume with respect to the measure induced by the restriction of $-B$ to $\mathrm{k} \cap \mathrm{m}^{\perp}$. Actually, this will follow from Proposition 10.1.17 in [He]. Indeed, let $d_{\mathrm{K}} x$ be the measure on $X$ induced by the restriction $\left.B_{\mathrm{K}}\right|_{\mathrm{p}}$ of the Killing form. So

$d x=(2 p+8 q)^{-n / 2} d_{\mathrm{K}} x$. Let $d_{\mathrm{K}} \bar{g}$ be the measure on $X$ induced by the Haar measure $d_{\mathrm{K}} g$ on $G$ such that $d_{\mathrm{K}} g=\gamma(a) d k_{1} d_{\mathrm{K}} a d k_{2}$ on $G^{+}=K A^{+} K$, where $d_{\mathrm{K}} a$ is the measure on $A$ induced by the Killing form $B_{\mathrm{K}}$. Now by Proposition 10.1.17 in [He] we have $d_{\mathrm{K}} x=\zeta_{\mathrm{K}} 2^{-p-q} d_{\mathrm{K}} \bar{g}$. Here $\zeta_{\mathrm{K}}=$ $\operatorname{vol}_{\mathrm{K}}(K / M)$, the volume computed with respect to the measure induced by the restriction of $-B_{\mathrm{K}}$ to $\mathrm{k} \cap \mathrm{m}^{\perp}$. This relationship between $d_{\mathrm{K}} x$ and $d_{\mathrm{K}} g$ implies that $d x=\zeta 2^{-p-q} d \bar{g}$, using that $d_{\mathrm{K}} a_{t}=\sqrt{2 p+8 q} d t$, and $\zeta=$ $(1 / \sqrt{2 p+8 q})^{\operatorname{dim}(\mathrm{k} / \mathrm{m})} \zeta_{\mathrm{K}}=(2 p+8 q)^{-(p+q) / 2} \zeta_{\mathrm{K}}=(2 p+8 q)^{(1-n) / 2} \zeta_{\mathrm{K}}$.

We wish to point out that in [MW], beginning of Section 5, the factor $2^{-p-q}$ is missing in the right hand side of the formula relating the measures $d x$ and $d \bar{g}$.

\section{The Resolvent of the LAPlacian}

The discrete subgroup $\Gamma \subset G$ with cofinite covolume satisfies the condition on p. 16 of [Langl]. (See Theorems 0.6 and 0.7 in [GR].) This allows us to use the well known spectral theory for $L^{2}(\Gamma \backslash G)$. On $\Gamma \backslash X$ we use the measure induced by the measure $d x$ on $X$.

Let $\Delta$ be the Laplacian on $X$ (or $\Gamma \backslash X$ ). We identify $-\Delta$ with the Casimir element $C$ of $G$, with respect to the form $B$. One has a spectral decomposition

$$
L^{2}(\Gamma \backslash X)=L_{d}^{2}(\Gamma \backslash X) \oplus^{\perp} L_{c}^{2}(\Gamma \backslash X)
$$

where the spectrum of $C$ is discrete (resp. continuous) in $L_{d}^{2}(\Gamma \backslash X)$ (resp. $\left.L_{c}^{2}(\Gamma \backslash X)\right)$. We fix a complete orthonormal set $\left\{\varphi_{j}\right\}_{j \geq 0}$ of real valued eigenfunctions of $C$ in $L_{d}^{2}(\Gamma \backslash X)$, with eigenvalues $\lambda_{j}=\rho^{2}-\nu_{j}^{2}$ arranged in increasing order, with $\operatorname{Re} \nu_{j} \geq 0$. So $\varphi_{0}$ is constant, and the other eigenvalues 
are positive. The explicit determination of such a basis is an open problem, even in the simplest cases. By a fundamental theorem of Langlands, $L_{c}^{2}(\Gamma \backslash X)$ is generated by eigenpackets of Eisenstein series, one of these for each cusp of $\Gamma$.

We note that the $\varphi_{j}$ here coincide with the $\varphi_{j}$ in $[\mathrm{MW}$, Section 5 , which differ from the $\varphi_{j}$ in $\left[\mathrm{MW}\right.$, Section 4 , by a factor $\zeta^{-\frac{1}{2}} 2^{\frac{p+q}{2}}$, since they are an orthonormal basis of $L_{d}^{2}(\Gamma \backslash X)$ with respect to the Riemannian measure $d x$ (and not $d \bar{g})$.

We now recapitulate some results on powers of the resolvent of $C$ on $X$ and $\Gamma \backslash X$.

Up to a meromorphic factor $s(\nu)$, the resolvent of $C$ on $X$ is given by convolution with a $K$-biinvariant function $Q_{\nu}$, real analytic on $G \backslash K$. The following result summarizes the main properties of $Q_{\nu}$ (see [MW] $\S 1, \S 2$ ).

Theorem 3.1. Let $\operatorname{Re} \nu \geq 0$. There exists a function $Q_{\nu} \in C^{\infty}\left(K \backslash G^{+} / K\right)$ such that

(i) $C Q_{\nu}=\lambda(\nu) Q_{\nu}$ on $G^{+}$, with $\lambda(\nu)=\rho^{2}-\nu^{2}$. Furthermore, if $g \in$ $G^{+}$, the map $\nu \rightarrow Q_{\nu}(g)$ is holomorphic for $\operatorname{Re} \nu \geq 0$ and admits a meromorphic continuation to $\mathrm{C}$.

(ii) $\varphi_{\nu}=c(-\nu) Q_{\nu}+c(\nu) Q_{-\nu}$. Here $\varphi_{\nu}$ is the zonal spherical function and $c(\nu)$ denotes Harish-Chandra's c-function (normalized as in $\S 1$, (3) of $[\mathrm{MW}]$, i.e. so that $c(\rho)=1)$.

(iii) If $n=2$, then $Q_{\nu}\left(a_{t}\right) \sim d(\nu)|\log (t)|$ as $t \downarrow 0$ and $Q_{\nu}\left(a_{t}\right) \sim d(\nu) t^{2-n}$ otherwise, with $d(\nu)$ meromorphic in C. Also, $Q_{\nu}\left(a_{t}\right) \sim a_{t}^{-(\nu+\rho)}$ as $t \rightarrow+\infty$. Moreover, there is an expansion

$$
Q_{\nu}(a)=a^{-(\nu+\rho)} \sum_{j=0}^{\infty} a_{j}(\nu) a^{-2 j \alpha}
$$

where $a_{0}(\nu)=1$ and $a_{j}(\nu)$ are rational functions, holomorphic for $\operatorname{Re} \nu \geq 0$, and uniformly bounded on vertical strips. This series is uniformly convergent for $a^{\alpha} \geq T$, for a sufficiently large positive constant $T$.

(iv) If $Q_{\nu}$ is defined then $Q_{\nu} \in L_{l o c}^{1}(G)$. Furthermore, for $\operatorname{Re} \nu>\rho$, we have $Q_{\nu} \in L^{p}(G)$, for $1 \quad p<2\left(Q_{\nu} \notin L^{2}(G)\right.$, if $\left.n>3\right)$.

If $r \in \mathrm{N}, r \geq 1$, let $Q_{r, \nu}=Q_{\nu} * \ldots * Q_{\nu} \quad(r$ times $)$. If $\operatorname{Re} \nu>$ $\rho, r>n / 4$, then $Q_{r, \nu} \in L^{p}(G)$, for $1 \quad p \quad 2+\varepsilon$, for some $\varepsilon>0$. Furthermore,

$$
(C-\lambda(\nu))^{r} Q_{r, \nu}=s(\nu)^{r} \delta
$$

with $s(\nu)=-2 \nu c(\nu)$ and $\delta(f)=f(1)$ for $f \in C(G)$.

The next theorem from [MW] shows that the $r$ times iterated resolvent on $\Gamma \backslash X$ has a kernel as well.

Theorem 3.2. Let $r \in \mathrm{N}^{+}$. 
(i) Let $[x]$ and $[y]$ be distinct points of $\Gamma \backslash X$, and let $\operatorname{Re} \nu>\rho$. The sum $\mathbf{P}_{r, \nu}(x, y)=s(\nu)^{-r} \sum_{\gamma \in \Gamma} Q_{r, \nu}\left(x^{-1} \gamma y\right)$ converges absolutely and defines $\mathbf{P}_{r, \nu}(x, y)$ holomorphic in $\nu$ and smooth in $x$ and $y$ in the complement of the diagonal on $(\Gamma \backslash X) \times(\Gamma \backslash X)$.

(ii) $(C-\lambda(\nu))^{r} \mathbf{P}_{r, \nu}(x, \cdot)=\zeta 2^{-p-q} \delta_{x}$, with $\delta_{x} f=f(x)$ for $f \in C_{c}^{\infty}(\Gamma \backslash X)$ and $\operatorname{Re} \nu>\rho$.

(iii) $\mathbf{P}_{r, \nu}(x, \cdot)$ admits a meromorphic continuation to $\mathbf{C}$, as a distribution. The only singularities in $\operatorname{Re} \nu \geq 0, \nu \neq 0$, occur at points $\mu$ such that $\lambda(\mu)$ is an eigenvalue of $C$ in $\overline{L^{2}}(\Gamma \backslash X)$, and have principal part

$$
\sum_{j, \nu_{j}=\mu}\left(\nu_{j}^{2}-\nu^{2}\right)^{-r} \zeta 2^{-p-q} \varphi_{j}(x) \varphi_{j}
$$

Proof. See [MW], $\S 3$, and Theorem 4.5, taking into account, for (ii) and (iii), the discussion on normalizations of measures at the end of Section 2. Note in particular, that we use in Part ii) the measure $d x$ to identify functions with distributions. Also, the proof of Theorem 3.5 in [MW], implies that (ii) holds for $\operatorname{Re} \nu>\rho$.

We now expand several results from [MW, Section 3] in the case $r=1$ that will be used in this paper.

We write $\mathbf{P}_{\nu}$ instead of $\mathbf{P}_{1, \nu}$.

In the domain $\operatorname{Re} \nu>\rho$ we have $\mathbf{P}_{\nu}(x, y)=\tilde{\mathbf{P}}_{\nu}(x, y)+\mathbf{P}_{\nu}^{\prime}(x, y)$, with

$$
\begin{aligned}
& \tilde{\mathbf{P}}_{\nu}(x, y)=s(\nu)^{-1} \sum_{\gamma \in \Gamma} \beta\left(x^{-1} \gamma y\right) Q_{\nu}\left(x^{-1} \gamma y\right) \\
& \mathbf{P}_{\nu}^{\prime}(x, y)=s(\nu)^{-1} \sum_{\gamma \in \Gamma}\left(1-\beta\left(x^{-1} \gamma y\right)\right) Q_{\nu}\left(x^{-1} \gamma y\right)
\end{aligned}
$$

where $\beta$ is a smooth $K$-biinvariant function on $G$, such that $\beta\left(a_{t}\right)=0$ for $|t|<T$, and $\beta\left(a_{t}\right)=1$ for $|t|>T+1$. In particular, $\beta Q_{\nu}$ is smooth. Later on we shall need to take $T$ as in Part (iii) of Proposition 3.1.

In this way, we obtain $\tilde{\mathbf{P}}_{\nu}$ as a function in $C^{\infty}((\Gamma \backslash X) \times(\Gamma \backslash X))$ for $\operatorname{Re} \nu>$ $\rho$. The remaining sum is locally finite in $x, y$, and defines $\mathbf{P}_{\nu}^{\prime}$ as a smooth function outside the diagonal of $(\Gamma \backslash X) \times(\Gamma \backslash X)$, meromorphic in $\nu \in \mathbf{C}$, holomorphic on $\operatorname{Re} \nu \geq 0$.

Proposition 3.3. $\mathbf{P}_{1, \nu}=\mathbf{P}_{\nu}$ is a smooth function on $(\Gamma \backslash X) \times(\Gamma \backslash X)$, outside the diagonal, for all $\nu$ where it is holomorphic. It is holomorphic in the set $\{\mu: \mu \neq 0, \operatorname{Re} \mu \geq 0\}$, except for possible simple poles that may occur only if $\lambda(\mu)$ is in the discrete spectrum.

$\tilde{\mathbf{P}}_{\nu}$ is a smooth function on $(\Gamma \backslash X) \times(\Gamma \backslash X)$, for all $\nu$ at which it is holomorphic. In the closed right half plane, it has the same singularities as $\mathbf{P}_{\nu}$. The residue of $\nu \mapsto \tilde{\mathbf{P}}_{\nu}(x, y)$ at $\nu=\mu \neq 0$, Re $\mu \geq 0$ is

$$
\frac{-\zeta 2^{-p-q}}{2 \mu} \sum_{j, \nu_{j}=\mu} \varphi_{j}(x) \varphi_{j}(y),
$$


for each $(x, y)$. If $[x] \neq[y]$, then this is also the residue of $\nu \mapsto \mathbf{P}_{\nu}(x, y)$.

Proof. Once Theorem 4.5 of [MW] has given the meromorphic continuation of $\mathbf{P}_{\nu}(x, \cdot)$, we obtain also the meromorphic continuation to $\mathbf{C}$ of $\tilde{\mathbf{P}}_{\nu}(x, \cdot)$ in the sense of distributions, with the same singularities as $\mathbf{P}_{\nu}$ in $\operatorname{Re} \nu \geq 0$. Proposition 4.6 of [MW] implies that this distribution is given by a smooth function on $(\Gamma \backslash X) \times(\Gamma \backslash X)$ for each value of $\nu$ with $\operatorname{Re} \nu \geq 0$ where it is holomorphic.

Theorem 4.1 in [MW] gives for $r>\frac{n}{4}$ and $\operatorname{Re} \nu>\rho$ a family $(\nu, x) \mapsto$ $\mathbf{P}_{r, \nu}(x, \cdot)$ of elements of $L^{2}(\Gamma \backslash X)$. The estimate in Theorem 4.2 is used in Proposition 4.3 to obtain the meromorphic continuation as a function of $\nu$. Moreover, the uniformity in Theorem 4.2 implies that $(\nu, x) \mapsto \mathbf{P}_{r, \nu}(x, \cdot)$ is continuous in $(\nu, x)$ outside the singularities. Furthermore, at $\nu=\mu$ with $\operatorname{Re} \mu \geq 0, \mu \neq 0$, the singularity is determined by the finitely many terms in the discrete sum corresponding to the $\nu_{j}$ such that $\nu_{j}^{2}=\mu^{2}$.

This family of $L^{2}$-elements determines a family of distributions on $X$. For each test function $\psi$ on $X$, we form $P_{\psi}: y \mapsto \sum_{\gamma} \overline{\psi(\gamma y)}$ in $L^{2}(\Gamma \backslash X)$ and define $\mathbf{P}_{r, \nu}(x ; \psi):=\left\langle\mathbf{P}_{r, \nu}(x, \cdot), P_{\psi}\right\rangle$. This result is meromorphic in $\nu$, and continuous in $(x, \nu)$ outside the singularities. In particular, for $r>\frac{n}{4}$ we have

$$
\mathbf{P}_{r, \nu}(x ; \psi)=\frac{\zeta 2^{-p-q}}{\left(\mu^{2}-\nu^{2}\right)^{r}} \sum_{j, \nu_{j}^{2}=\mu^{2}} \varphi_{j}(x)\left\langle\varphi_{j}, P_{\psi}\right\rangle+\mathbf{R}_{r, \nu}(x ; \psi),
$$

where $\mathbf{R}_{r, \nu}(x)$ is a distribution and $(\nu, x) \mapsto \mathbf{R}_{r, \nu}(x ; \psi)$ is continuous and holomorphic at $\nu=\mu$. In fact, $\mathbf{R}_{r, \nu}$ is given by the remaining part of the expansion in Theorem 4.1, to which we can apply the estimate in Theorem 4.2.

Furthermore, if $\operatorname{Re} \nu>\rho$ we have $\left(C_{y}-\lambda(\nu)\right) \mathbf{P}_{r, \nu}(x, \cdot)=\mathbf{P}_{r-1, \nu}(x, \cdot)$ for $r \geq 2$. Since for large $r$ we have already $\mathbf{P}_{r, \nu}(x, \cdot)$ as a meromorphic family of distributions, this gives successively the extension of $\mathbf{P}_{r, \nu}(x, \cdot)$ as a meromorphic family for those values of $r$ for which Theorem 4.2 does not apply. Moreover, if we define $\mathbf{R}_{r, \nu}(x)$ as the distributional derivative $\left(C_{y}-\lambda(\nu)\right) \mathbf{R}_{r+1, \nu}(x)$, the fact that $\varphi_{j}$ is an eigenfunction of $C$ shows that (4) remains valid for any $r \geq 1$. So $\mathbf{R}_{r, \nu}(x ; \psi)=\mathbf{R}_{r+1, \nu}\left(x ;\left(C_{y}-\lambda(\nu)\right) \psi\right)$ is continuous in $(\nu, x)$ and holomorphic at $\nu=\mu$.

In particular, we have $\mathbf{P}_{1, \nu}(x ; \psi)=\mathbf{P}_{r, \nu}\left(x ;\left(C_{y}-\lambda(\nu)\right)^{r-1} \psi\right)$. The expansion in Theorem 4.1 shows that $\mathbf{P}_{1, \nu}(x ; \psi)$ is continuous in $(\nu, x)$ and meromorphic in $\nu$, and that it is, uniformly for $x$ in compact sets, bounded by the supremum norm of $\left(C_{y}-\lambda(\nu)\right)^{r-1} \psi$ times the volume of the support of $\psi$. So $\mathbf{P}_{1, \nu}(x, \cdot)$ is a family of distributions of order at most $2 r-2$. The same holds for $\mathbf{R}_{1, \nu}(x)$, but here we have holomorphy at $\nu=\mu$ as well.

$\mathbf{P}_{1, \nu}^{\prime}(x, y)$ is well defined for $x \neq \gamma y$ for all $\gamma \in \Gamma$. The asymptotics in part iv) of Theorem 1.1, and the formula for the Haar measure on page 667 make clear that $y \mapsto \mathbf{P}_{1, \nu}^{\prime}(x, y)$ is locally integrable. So we have a family of distributions $(\nu, x) \mapsto \mathbf{P}_{1, \nu}^{\prime}(x, \cdot)$ that turns out to be continuous in $(\nu, x)$ and holomorphic on $\operatorname{Re} \nu \geq-\varepsilon$ for some $\varepsilon>0$. 
Thus we have the meromorphy in $\nu$ and the continuity in $(\nu, x)$ of the difference of distributions $\tilde{\mathbf{P}}_{1, \nu}(x, \cdot):=\mathbf{P}_{1, \nu}(x, \cdot)-\mathbf{P}_{1, \nu}^{\prime}(x, \cdot)$, and also of $\tilde{\mathbf{R}}_{1, \nu}(x, \cdot):=\mathbf{R}_{1, \nu}(x, \cdot)-\mathbf{P}_{1, \nu}^{\prime}(x, \cdot)$. For each test function $\psi$ we have

$$
\tilde{\mathbf{P}}_{1, \nu}(x ; \psi)=\frac{\zeta 2^{-p-q}}{\mu^{2}-\nu^{2}} \sum_{j} \varphi_{j}(x) \int_{X} \varphi_{j}(y) \psi(y) d y+\tilde{\mathbf{R}}_{1, \nu}(x ; \psi) .
$$

Integrating the first term with respect to $x$ gives a distribution on $X \times X$, given by $\frac{\zeta 2^{-p-q}}{\mu^{2}-\nu^{2}} \sum_{j} \varphi_{j}(x) \varphi_{j}(y)$. By the properties of $\tilde{\mathbf{R}}_{r, \nu}(x ; \psi)$ mentioned previously, the second term gives a distribution $\tilde{\mathbf{R}}_{1, \nu}(\cdot, \cdot)$ on $X \times X: \psi \mapsto$ $\int_{X} \tilde{\mathbf{R}}_{1, \nu}(x ; \psi(x, \cdot)) d x$.

Let $U$ be a simply connected open neighborhood of $\mu$ in which $\mu$ is the only singular point and with the condition that $U$ has a non-empty intersection with the region of absolute convergence $\{\nu: \operatorname{Re} \nu>\rho\}$. Integrating over $U$ as well, we obtain a distribution $\tilde{r}$ on $U \times X \times X$ :

$$
\tilde{r}(\psi)=\int_{U} \int_{X} \tilde{\mathbf{R}}_{1, \nu}(x ; \psi(\nu, x, \cdot)) d x \frac{d \nu d \bar{\nu}}{-2 i} .
$$

For $\nu \in U, \nu \neq \mu$, we can define $\tilde{\mathbf{P}}_{1, \nu}(\cdot, \cdot)$ as a distribution on $X \times X$ in a similar way. The reasoning in the proofs of Lemma 3.2 and Proposition 4.6 shows that it satisfies

$$
\left(C_{x}-\lambda(\nu)+C_{y}-\lambda(\nu)\right) \tilde{\mathbf{P}}_{1, \nu}(\cdot, \cdot)=F_{\nu},
$$

for some smooth function $F_{\nu}$ on $X \times X$, which is holomorphic for $\nu \in U \backslash\{\mu\}$.

Similarly, $\sum_{j} \varphi_{j} \backslash \varphi_{j}$ satisfies a differential equation of the same type, and so does $\tilde{\mathbf{R}}_{1, \nu}$ :

$$
\left(C_{x}-\lambda(\nu)+C_{y}-\lambda(\nu)\right) \tilde{\mathbf{R}}_{1, \nu}(\cdot, \cdot)=\tilde{F}_{\nu},
$$

for some smooth function $F_{\nu}$ on $X \times X$ depending meromorphically on $\nu$. But the holomorphy of $\tilde{\mathbf{R}}_{1, \nu}(\cdot, \cdot)$ on $U$ implies that $\tilde{F}_{\nu}$ is holomorphic on $U$ as a distribution on $X \times X$.

We now apply the differential operator $\partial_{\nu} \partial_{\bar{\nu}}$ to $\tilde{r}$. For a test function $\psi$ on $U \times X \times X$, we find

$$
\begin{gathered}
\partial_{\nu} \partial_{\bar{\nu}} \tilde{r}(\psi)=\int_{U} \int_{X} \int_{X} \tilde{\mathbf{R}}_{r, \nu}(x, y)\left(C_{y}-\lambda(\nu)\right) \partial_{\bar{\nu}} \partial_{\nu} \psi(\nu, x, y) d y d x \frac{d \nu d \bar{\nu}}{-2 i} \\
\quad=\int_{X} \int_{X} \int_{U} \tilde{\mathbf{R}}_{r, \nu}(x, y) \partial_{\bar{\nu}} \partial_{\nu} \psi_{1}(\nu, x, y) \frac{d \nu d \bar{\nu}}{-2 i} d x d y
\end{gathered}
$$

where $\psi_{1}$ is another test function (obtained by interchanging the differentiations) and $\tilde{\mathbf{R}}_{r, \nu}(x, y)$ given by a representation as in Theorem 4.1 , but with a few terms omitted. As $\tilde{\mathbf{R}}_{r, \nu}$ is holomorphic on $U$, we find zero after partial integration in the $\nu$-variable. So $\partial_{\nu} \partial_{\bar{\nu}} \tilde{r}=0$.

Let us now consider the elliptic operator $\mathcal{L}=-\partial_{\nu} \partial_{\bar{\nu}}+C_{x}-\lambda(\nu)+C_{y}-$ $\lambda(\nu)+$ on $U \times X \times X$. We have $\mathcal{L} \tilde{r}=\tilde{F}_{\nu}$ in distribution sense on $U \times X \times X$. But $\tilde{F}_{\nu}$ is smooth in all three variables jointly. The conclusion is that $\tilde{r}$ is 
given by a smooth function on $U \times X \times X$ which is holomorphic in $\nu \in U$. We denote this function by $\tilde{r}(\nu, x, y)$ as well.

Finally, $p:(\nu, x, y) \mapsto \frac{\zeta 2^{-p-q}}{\mu^{2}-\nu^{2}} \sum_{j} \varphi_{j}(x) \varphi_{j}(y)+\tilde{r}(\nu, x, y)$ determines a distribution on $U \times X \times X$. This function is smooth outside its singularities, and its principal part is explicitly given.

As a distribution on $U \times X \times X$, it is the meromorphic continuation of the function $\tilde{\mathbf{P}}_{1, \nu}(x, y)$ given by a convergent series for $\operatorname{Re} \nu>\rho$. So, the distribution on $X$ obtained by integrating against $p(\nu, x, \cdot)$ is the same as the distribution $\tilde{\mathbf{P}}_{1, \nu}(x, \cdot)$. This completes the proof.

We now prove an estimate which will play a fundamental role in the sequel.

Lemma 3.4. If $r \in \mathrm{N}^{+}$, then $(C-\lambda(\nu))^{r} \tilde{\mathbf{P}}_{\nu}(x, \cdot) \in L^{2}(\Gamma \backslash X)$. Let $s(\nu)=$ $-2 \nu c(\nu)$, fix $\sigma, \sigma^{\prime}$ with $0<\sigma<\sigma^{\prime}$, and let $-\subset X$, a compact subset. Then there is $C_{-, \sigma, \sigma^{\prime}} \geq 0$ such that

$$
\left\|s(\nu)(C-\lambda(\nu))^{r} \tilde{\mathbf{P}}_{\nu}(x, \cdot)\right\|_{2} \quad C_{-, \sigma, \sigma^{\prime}}(1+|\nu|)^{r}
$$

for all $x \in-$ and $\nu$ in $S_{\sigma, \sigma^{\prime}}:=\left\{\begin{array}{lll}\nu: \sigma & \operatorname{Re} \nu & \sigma^{\prime}\end{array}\right\}$. $G$.

We give a proof that does not use the fact that $\Gamma$ has finite covolume in

Proof. We claim that $s(\nu)(C-\lambda(\nu))^{r} \tilde{\mathbf{P}}_{\nu}(x, \cdot)$ can be written as a finite sum of functions of the form

$$
\sum_{\gamma \in \Gamma} Y_{i_{1}} \ldots Y_{i_{k}} \beta\left(x^{-1} \gamma y\right) \cdot X_{j_{1}} \ldots X_{j_{h}} Q_{\nu}\left(x^{-1} \gamma y\right)
$$

with $r \quad k \quad 2 r, 0 \quad h \quad r$, and all $Y_{i}, X_{j} \in g$. Indeed if $r=1$ and $\operatorname{Re} \nu>\rho$, since $(C-\lambda(\nu)) Q_{\nu}=0$, where defined, we get that $s(\nu)(C-\lambda(\nu)) \tilde{\mathbf{P}}_{\nu}(x, y)$ is a sum of terms of the form

$$
\sum_{\gamma \in \Gamma} X_{i} X_{j} \beta\left(x^{-1} \gamma y\right) \cdot Q_{\nu}\left(x^{-1} \gamma y\right)+\sum_{\gamma \in \Gamma} X_{i}^{\prime} \beta\left(x^{-1} \gamma y\right) \cdot X_{j}^{\prime} Q_{\nu}\left(x^{-1} \gamma y\right) .
$$

Now, if we apply $C-\lambda(\nu)$ to an expression as in (5), this will introduce either two more derivatives on $\beta$, or one derivative on $\beta$ and one on $Q_{\nu}$, hence the inductive step from $r$ to $r+1$ follows.

We also note that if $X \in \mathrm{g}$, then $X \beta$ is compactly supported on $G$, hence, locally in $x, y$, the sums in (5) have finitely many non-zero terms. Hence the expansion (5) is valid for $\nu \in \mathrm{C}$, outside the singularities of $Q_{\nu}$. This implies in particular that if $r \geq 1, x, y \in G, s(\nu)(C-\lambda(\nu))^{r} \tilde{\mathbf{P}}_{\nu}(x, y)$ has a meromorphic continuation to $\bar{C}$, with possible poles at the poles of $Q_{\nu}$, in particular, it is holomorphic for $\operatorname{Re} \nu \geq 0$. Furthermore, this continuation is given by a smooth function on $(\Gamma \backslash X) \times(\Gamma \backslash X)$.

We now prove that for any $X_{1}, \ldots, X_{h} \in \mathrm{g}$, there exists $C=C_{\sigma, \sigma^{\prime}, X_{1}, \ldots, X_{h}}$, such that

$$
X_{1} \ldots X_{h} Q_{\nu}(g) \quad C|\nu|^{h}
$$


uniformly for $g$ in compact subsets of $\left\{k_{1} a_{t} k_{2}: k_{1}, k_{2} \in K, t>T\right\}$ with $T$ as in Part (iii) of Proposition 3.1, and $\nu$ in the strip $\sigma<\operatorname{Re} \nu<\sigma^{\prime}$.

If we set $\psi(x)=\alpha(\log a(x))$, using the expansion (3) for $Q_{\nu}$, we see that the left hand side of (6) equals

$$
\begin{aligned}
& \frac{d}{d t_{h \mid 0}} \ldots \frac{d}{d t_{1 \mid 0}} Q_{\nu}\left(g \exp \left(t_{1} X_{1}\right) \ldots \exp \left(t_{h} X_{h}\right)\right) \\
= & \sum_{j \geq 0} a_{j}(\nu) \frac{d}{d t_{h \mid 0}} \ldots \frac{d}{d t_{1 \mid 0}} e^{-(\nu+\rho+2 j) \psi\left(g \exp \left(t_{1} X_{1}\right) \ldots \exp \left(t_{h} X_{h}\right)\right)}
\end{aligned}
$$

Now an inductive argument shows that

$$
\frac{d}{d t_{h} \mid 0} \ldots \frac{d}{d t_{1} \mid 0} e^{-(\nu+\rho+2 j) \psi\left(g \exp \left(t_{1} X_{1}\right) \ldots \exp \left(t_{h} X_{h}\right)\right)}
$$

is a sum of terms of the form

$$
(-(\nu+\rho+2 j))^{l} e^{-(\nu+\rho+2 j) \psi(g)} X_{I_{1}} \psi(g) X_{I_{2}} \psi(g) \ldots X_{I_{l}} \psi(g)
$$

where $I_{1} \cup \ldots \cup I_{l}=\{1, \ldots, h\}$ and $X_{I_{j}} \psi(g)=X_{i_{j, 1}} \ldots X_{i_{j, l}} \psi(g)$, if $I_{j}=$ $\left\{i_{j, 1}, \ldots, i_{j, l_{j}}\right\}$. We have $1 \quad l \quad h$ and $\sum i_{j, l_{j}}=h$. Hence (7) can be estimated by

$$
|\nu+\rho|^{h} \sum_{l=0}^{h}\left|\psi_{l}(g)\right|\left(\sum_{j \geq 0}\left|a_{j}(\nu)\right|\left|\frac{(\nu+\rho+2 j)^{l}}{(\nu+\rho)^{h}}\right| a(g)^{-(\operatorname{Re} \nu+\rho+2 j) \alpha}\right)
$$

where each $\psi_{l}(g)$ is a finite sum of functions of the form $X_{I_{1}} \psi(g) \ldots X_{I_{l}} \psi(g)$.

Since $l \quad h,(8)$ together with the uniform convergence of (3) implies that the series in (7) converges uniformly for $a(g) \geq T>0$, and $\nu$ on the strip $S_{\sigma, \sigma^{\prime}}$, with sum uniformly bounded on $S_{\sigma, \sigma^{\prime}}$ by $C|\nu|^{h}$. This implies (6).

We now claim that the lemma follows from (5) and (6). We denote $q_{\nu}(g)=$ $Y_{i_{1}} \ldots Y_{i_{k}} \beta(g) \cdot X_{j_{1}} \ldots X_{j_{h}} Q_{\nu}(g)$. The properties of the cut-off function $\beta$ imply that $q_{\nu}$ has compact support - ' in $G$. In particular, this support is contained in the region where (6) holds.

We denote left translation by $x \in G$, by $L_{x} f(g)=f\left(x^{-1} g\right)$. If $x \in$ - , compact, all the $L_{x} q_{\nu}$ have support contained in the compact set - - ' $=$ - "'. For each $x \in$ - , the average of $L_{x} q_{\nu}$ over $\Gamma$ has compact support in $\Gamma \backslash G$ included in $\pi\left(-{ }^{\prime \prime}\right), \pi: G \mapsto \Gamma \backslash G$, the quotient map. Now

$$
\begin{aligned}
& \int_{\Gamma \backslash G}\left|\sum_{\gamma} q_{\nu}\left(x^{-1} \gamma y\right)\right|^{2} d y=\int_{\pi\left(-{ }^{\prime \prime}\right)}\left|\sum_{\gamma} q_{\nu}\left(x^{-1} \gamma y\right)\right|^{2} d y \\
& \left.\operatorname{vol} \pi\left(-{ }^{\prime \prime}\right) \sup _{y \in-} "|| \sum_{\gamma} q_{\nu}\left(x^{-1} \gamma y\right)\right|^{2} \\
& \operatorname{vol} \pi\left(-{ }^{\prime \prime}\right)\left\|q_{\nu}\right\|_{\infty}{ }^{2}\left|\Gamma \cap--^{\prime}{ }^{\prime}{ }^{\prime \prime-1}\right|^{2}
\end{aligned}
$$

This clearly implies the assertion in the lemma in the light of (5) and (6). 
In the sequel, we shall use the spectral function of the Laplacian on $\Gamma \backslash X$, which we define by

$$
\begin{aligned}
e(x, y ; \lambda):=\sum_{\lambda\left(\nu_{j}\right) \lambda} \varphi_{j}(x) \varphi_{j}(y) & \quad+\sum_{\ell} c_{Q_{\ell}} \int_{\mu \in \mathrm{R}, \rho^{2}+\mu^{2} \quad \lambda} \overline{E\left(Q_{\ell}, i \mu, x\right)} E\left(Q_{\ell}, i \mu, y\right) d \mu \\
& =\int_{t=0-}^{\lambda} d(e(x, y ; t)),
\end{aligned}
$$

where $x, y \in X, \lambda \geq 0$. The $c_{Q_{\ell}}$ are the constants needed in the spectral expansion in eigenfunctions of the Casimir operator. They are proportional to the $c_{j}$ in Theorem 4.1 of [MW].

Note that $e(x, y ; \lambda)$ is $\Gamma$-invariant in $x$ as well as in $y$. We shall use the following result:

Lemma 3.5. For each $x \in X$ we have

$$
e(x, x ; \lambda)=O\left(\lambda^{n / 2}\right) \quad(\lambda \rightarrow \infty),
$$

and, if $\lambda \quad \lambda_{1} 2 \lambda$ :

$$
e\left(x, x ; \lambda_{1}\right)-e(x, x ; \lambda)=O\left(\left(\lambda_{1}-\lambda\right) \lambda^{n / 2-1}+\lambda^{(n-1) / 2}\right) \quad(\lambda \rightarrow \infty) .
$$

The estimate in (10) is uniform for $x$ in compact subsets of $\Gamma \backslash X$.

The proof shows that for torsion free $\Gamma$ we also have uniformity of the estimate (11).

Proof. Borel shows in Proposition 17.6 and Corollaire 17.7 of [Bo], that any finitely generated subgroup of $\operatorname{GL}(n, K)$ over a field $K$ of characteristic zero has a "net" subgroup of finite index. The concept net implies torsion free. To get $G$ inside a suitable general linear group, we take it in its adjoint form. This does not change the problem, as we are only interested in the quotient $X=G / K$. Going over to a covering involves a finite center, which is contained in $K$. This does not change the spectral function. This means that $\Gamma$ has a torsion-free subgroup $\Gamma^{\prime}$ of finite index. We can suppose $\Gamma^{\prime}$ to be normal in $\Gamma$.

We shall derive the lemma from a result of Hörmander, see [Hö], Theorem 5.1. It implies, since $\Gamma^{\prime}$ is torsion free, that there is a smooth function $\varphi$ on $X$ such that the spectral function satisfies

$$
e_{\Gamma^{\prime}}(x, x ; \lambda)=\varphi(x) \lambda^{n / 2}+O\left(\lambda^{(n-1) / 2}\right) \quad(\lambda \rightarrow \infty),
$$

uniform for $x$ in compact subsets of $\Gamma^{\prime} \backslash X$, and

$$
e_{\Gamma^{\prime}}(x, y ; \lambda)=O\left(\lambda^{(n-1) / 2}\right) \quad(\lambda \rightarrow \infty),
$$

uniformly for $(x, y)$ in compact subsets of $\left(\Gamma^{\prime} \backslash X\right) \times\left(\Gamma^{\prime} \backslash X\right)$ that do not meet the diagonal. 
It follows that the lemma is clear in the case that $\Gamma$ has no torsion, even with uniformity in (11). We shall reduce the general case to the torsion-free case.

As above, let $\Gamma^{\prime}$ be a normal torsion-free subgroup of $\Gamma$, of finite index. The spectral function $e_{\Gamma^{\prime}}$ satisfies the assertions (12) and (13). The spectral function $e$ for the group $\Gamma$ is given by

$$
e(x, y ; \lambda)=\sum_{\gamma \in \Gamma^{\prime} \backslash \Gamma} e_{\Gamma^{\prime}}(\gamma x, y ; \lambda) .
$$

(This relation is based on the assumption that the invariant measures on $\Gamma^{\prime} \backslash X$ and $\Gamma \backslash X$ come from the same Haar measure on $G$.)

The Cauchy-Schwartz inequality implies

$$
\left|e_{\Gamma^{\prime}}(x, y ; \lambda)\right| \quad e_{\Gamma^{\prime}}(x, x ; \lambda)^{1 / 2} e_{\Gamma^{\prime}}(y, y ; \lambda)^{1 / 2}
$$

for all $x, y \in X$. For a fixed system $R$ of representatives of $\Gamma^{\prime} \backslash \Gamma$ and a compact set - $\subset X$, the union $\bigcup_{\gamma \in R} \gamma^{-}$is also compact. So (12) implies that $e(x, y ; \lambda)=O\left(\lambda^{n / 2}\right)$, uniform for $x$ and $y$ in compact sets in $X$. Thus we obtain assertion (10) uniformly on $\Gamma \backslash X$.

Let $x \in X$ be fixed, and denote by $\Gamma_{x}$ the subgroup of $\Gamma$ leaving $x$ fixed. Then

$$
e(x, x ; \lambda)=u(x) \sum_{\Gamma_{x} \backslash \Gamma} e_{\Gamma^{\prime}}(\gamma x, x ; \lambda),
$$

where $u(x)=\#\left(\left(\Gamma^{\prime} \cap \Gamma_{x}\right) \backslash \Gamma_{x}\right)=\# \Gamma_{x}$. From (12) and (13) it follows that

$$
e(x, x ; \lambda)=u(x) \varphi_{\Gamma^{\prime}}(x) \lambda^{n / 2}+O\left(\lambda^{(n-1) / 2}\right) \quad(\lambda \rightarrow \infty),
$$

where $\varphi_{\Gamma^{\prime}}$ is a smooth function on $X$. This gives (11) by a short computation. Note that $x \mapsto u(x) \varphi_{\Gamma^{\prime}}(x)$ need not be continuous on $X$.

Theorem 3.6. Let $\sigma, \varepsilon>0$, and let $x, y \in G$. There exists $C_{\sigma, \varepsilon}$ such that

$$
\left|s(\nu) \tilde{\mathbf{P}}_{\nu}(x, y)\right| \quad C_{\sigma, \varepsilon}|\operatorname{Im} \nu|^{\frac{n-1}{2}+\varepsilon}
$$

uniformly for $\nu \in S_{\sigma}=\left\{\begin{array}{lll}\nu: \sigma & \operatorname{Re} \nu & \rho+\sigma\end{array}\right\},|\operatorname{Im} \nu| \geq 1$.

In the case of torsion free $\Gamma$, this estimate can be shown to be uniform for $x$ and $y$ in compact sets.

Proof. Let $\operatorname{Re} \nu>\rho$. Lemma 3.2 in $[\mathrm{MW}]$ shows that $\tilde{\mathbf{P}}_{\nu}(x, \cdot) \in L^{s}(\Gamma \backslash X)$ for each $s>2$. The derivatives $\left(C_{y}-\lambda(\nu)\right)^{j} \tilde{\mathbf{P}}_{\nu}(x, y)$ have compact support in $y \in \Gamma \backslash X$ for $j \geq 1$. Theorem 4.7 in [MW] shows that if $r$ is any integer $r>n / 4$, there is an absolutely convergent representation:

$$
\begin{aligned}
& \widetilde{\mathbf{P}}_{\nu}(x, y)=\sum\left\langle(C-\lambda(\nu))^{r} \widetilde{\mathbf{P}}_{\nu}(x, \cdot), \varphi_{j}\right\rangle \frac{\varphi_{j}(y)}{\left(\nu_{j}^{2}-\nu^{2}\right)^{r}} \\
& \quad+\sum_{Q_{\ell}} c_{Q_{\ell}} \int_{-\infty}^{\infty}\left\langle(C-\lambda(\nu))^{r} \widetilde{\mathbf{P}}_{\nu}(x, \cdot), E\left(Q_{\ell}, i \mu, \cdot\right)\right\rangle \frac{E\left(Q_{\ell}, i \mu, y\right)}{\left(\mu^{2}+\nu^{2}\right)^{r}} d \mu
\end{aligned}
$$


valid for $\operatorname{Re} \nu>\rho$.

By applying the Cauchy-Schwartz inequality to the right hand side, we see that $s(\nu) \widetilde{\mathbf{P}}_{\nu}(x, y)$ is estimated in absolute value by

$$
\begin{aligned}
& \ll s(\nu)\left(\sum_{j}\left|\left\langle(C-\lambda(\nu))^{r} \widetilde{\mathbf{P}}_{\nu}(x, \cdot), \varphi_{j}\right\rangle\right|^{2}\right)^{1 / 2}\left(\sum_{j} \frac{\left|\varphi_{j}(y)\right|^{2}}{\left|\nu_{j}^{2}-\nu^{2}\right|^{2 r}}\right)^{1 / 2} \\
&+ s(\nu) \sum_{\ell} c_{Q_{\ell}}\left(\int_{-\infty}^{\infty}\left|\left\langle(C-\lambda(\nu))^{r} \widetilde{\mathbf{P}}_{\nu}(x, \cdot), E\left(Q_{\ell}, i \mu, \cdot\right)\right\rangle\right|^{2} d \mu\right)^{1 / 2} \\
& \cdot\left(\int_{-\infty}^{\infty} \frac{\left|E\left(Q_{\ell}, i \mu, y\right)\right|^{2}}{\left|\mu^{2}+\nu^{2}\right|^{2 r}} d \mu\right)^{1 / 2} \\
& \ll \quad(1+|\nu|)^{r}\left(\sum_{j} \frac{\left|\varphi_{j}(y)\right|^{2}}{\left|\nu_{j}^{2}-\nu^{2}\right|^{2 r}}+\sum_{\ell} c_{Q_{\ell}} \int_{-\infty}^{\infty} \frac{\left|E\left(Q_{\ell}, i \mu, y\right)\right|^{2}}{\left|\mu^{2}+\nu^{2}\right|^{2 r}} d \mu\right)^{1 / 2},
\end{aligned}
$$

with use of the uniform estimate on $S_{\sigma}$ for $\left\|s(\nu)(C-\lambda(\nu))^{r} \widetilde{\mathbf{P}}_{\nu}(x, \cdot)\right\|_{2}$ in Lemma 3.4.

Now we note that

$$
\begin{aligned}
\rho^{2} \quad \sum_{\lambda\left(\nu_{j}\right)} \frac{\left|\varphi_{j}(x)\right|^{2}}{\left(\lambda\left(\nu_{j}\right)+1\right)^{n / 2+\varepsilon}}+\sum_{\ell} c_{Q_{\ell}} \int_{-\sqrt{T-\rho^{2}}}^{\sqrt{T-\rho^{2}}} \frac{\left|E\left(Q_{\ell}, i \mu, x\right)\right|^{2}}{\left(\mu^{2}+\rho^{2}+1\right)^{n / 2+\varepsilon}} d \mu \\
=\int_{\rho^{2}-0}^{T} \frac{d(e(x, x ; \lambda))}{(\lambda+1)^{n / 2+\varepsilon}} \\
=\left.\frac{e(x, x ; \lambda)}{(\lambda+1)^{n / 2+\varepsilon}}\right|_{\rho^{2}-0} ^{T}+\left(\frac{n}{2}+\varepsilon\right) \int_{\rho^{2}}^{T} \frac{e(x, x ; \lambda)}{(\lambda+1)^{n / 2+1+\varepsilon}} d \lambda \ll T^{-\varepsilon},
\end{aligned}
$$

by $(10)$.

This implies the convergence of the estimate of $\tilde{\mathbf{P}}_{\nu}$ obtained above, for any $r \in \mathrm{R}, r>\frac{n}{4}$ :

$$
\begin{aligned}
& s(\nu) \tilde{\mathbf{P}}_{\nu}(x, y) \ll(1+|\nu|)^{r} \\
& \quad \cdot\left(\sum_{j} \frac{\left|\varphi_{j}(y)\right|^{2}}{\left|\nu_{j}^{2}-\nu^{2}\right|^{2 r}}+\sum_{\ell} c_{Q_{\ell}} \int_{-\infty}^{\infty} \frac{\left|E\left(Q_{\ell}, i \mu, y\right)\right|^{2}}{\left|\mu^{2}+\nu^{2}\right|^{2 r}} d \mu\right)^{1 / 2} .
\end{aligned}
$$

In the sequel, we shall use (11) to give an estimate for (15), uniform on vertical strips. We consider the following quantity, whose square root occurs in (15):

$$
S(\nu):=\sum_{j} \frac{\left|\varphi_{j}(x)\right|^{2}}{\left|\nu_{j}^{2}-\nu^{2}\right|^{2 r}}+\sum_{\ell} c_{Q_{\ell}} \int_{-\infty}^{\infty} \frac{\left|E\left(Q_{\ell}, i \mu, x\right)\right|^{2}}{\left|\mu^{2}+\nu^{2}\right|^{2 r}} d \mu
$$

with $r \in \mathrm{N}, r>\frac{n}{4}$ to be chosen later. We take apart $0<\nu_{j}<\rho$ for 1 $j \quad m$, corresponding to the exceptional eigenvalues. The other eigenvalues 
correspond to $\nu_{j}=i t_{j}, t_{j} \in \mathrm{R}$, for $j>m$. Also set $\nu=s+i t, s, t \in \mathrm{R}$. Thus, if $j>m,\left|\nu_{j}^{2}-\nu^{2}\right|^{2}=\left(s^{2}+\left(t_{j}-t\right)^{2}\right)\left(s^{2}+\left(t_{j}+t\right)^{2}\right)$. We note that it is sufficient to prove the estimate for $t>0$, so we may and will assume that $t_{j} \geq 0$ for all $j>m$. Fix $0<\vartheta<1$, to be determined later. We split $S(\nu)=S_{e}(\nu)+S_{1}(\nu)+S_{2}(\nu)$, where $S_{e}(\nu)=\sum_{j=1}^{m} \frac{\left|\varphi_{j}(x)\right|^{2}}{\left|\nu_{j}^{2}-\nu^{2}\right|^{2 r}}$, the contribution of the exceptional spectrum to (16), and

$$
\begin{aligned}
S_{1}(\nu)= & \sum_{\left|t_{j} \pm t\right| \geq t^{\vartheta}} \frac{\left|\varphi_{j}(x)\right|^{2}}{\left(s^{2}+\left(t_{j}-t\right)^{2}\right)^{r}\left(s^{2}+\left(t_{j}+t\right)^{2}\right)^{r}} \\
& +\sum_{\ell} c_{Q_{\ell}} \int_{|\mu \pm t| \geq t^{\vartheta}} \frac{\left|E\left(Q_{\ell}, i \mu, x\right)\right|^{2}}{\left(s^{2}+(\mu-t)^{2}\right)^{r}\left(s^{2}+(\mu+t)^{2}\right)^{r}} d \mu, \\
S_{2}(\nu)= & S(\nu)-S_{e}(\nu)-S_{1}(\nu) \\
= & \sum_{\left|t_{j}-t\right|<t^{\vartheta}} \frac{\left|\varphi_{j}(x)\right|^{2}}{\left(s^{2}+\left(t_{j}-t\right)^{2}\right)^{r}\left(s^{2}+\left(t_{j}+t\right)^{2}\right)^{r}} \\
& +\sum_{\left|t_{j}+t\right|<t^{\vartheta}} \frac{\left|\varphi_{j}(x)\right|^{2}}{\left(s^{2}+\left(t_{j}-t\right)^{2}\right)^{r}\left(s^{2}+\left(t_{j}+t\right)^{2}\right)^{r}} \\
& +\sum_{\ell} c_{Q_{\ell}} \int_{|\mu-t|<t^{\vartheta}} \frac{\left|E\left(Q_{\ell}, i \mu, x\right)\right|^{2}}{\left(s^{2}+(\mu-t)^{2}\right)^{r}\left(s^{2}+(\mu+t)^{2}\right)^{r}} d \mu \\
& +\sum_{\ell} c_{Q_{\ell}} \int_{|\mu+t|<t^{\vartheta}} \frac{\left|E\left(Q_{\ell}, i \mu, x\right)\right|^{2}}{\left(s^{2}+(\mu-t)^{2}\right)^{r}\left(s^{2}+(\mu+t)^{2}\right)^{r}} d \mu .
\end{aligned}
$$

We first estimate $S_{1}(\nu)$, uniformly on $S_{\sigma}$. We will use the following estimate: For $0<\alpha<1$, there exists $C_{\alpha}>0$ such that for $t, u \in \mathrm{R}^{+}$we have the following inequality:

$$
\left(1+(t+u)^{2}\right) \geq C_{\alpha}\left(1+t^{2}\right)^{\alpha}\left(1+u^{2}\right)^{1-\alpha},
$$

We obtain the following estimates:

$$
\begin{aligned}
S_{1}(\nu) \ll & \frac{1}{\left(1+t^{2 \vartheta}\right)^{r}}\left(\sum_{\left|t_{j} \pm t\right| \geq t^{\vartheta}} \frac{\left|\varphi_{j}(x)\right|^{2}}{\left(1+\left(t_{j}+t\right)^{2}\right)^{r}}\right. \\
& \left.+\sum_{\ell} c_{Q_{\ell}} \int_{|\mu \pm t| \geq t^{\vartheta}} \frac{\left|E\left(Q_{\ell}, i \mu, x\right)\right|^{2}}{\left(1+(\mu+t)^{2}\right)^{r}} d \mu\right) \\
\ll \alpha & \frac{1}{\left(1+t^{2}\right)^{r \vartheta+r(1-\alpha)}}\left(\sum_{j} \frac{\left|\varphi_{j}(x)\right|^{2}}{\left(1+t_{j}^{2}\right)^{r \alpha}}\right. \\
& \left.+\sum_{\ell} c_{Q_{\ell}} \int_{-\infty}^{\infty} \frac{\left|E\left(Q_{\ell}, i \mu, x\right)\right|^{2}}{\left(1+\mu^{2}\right)^{r \alpha}} d \mu\right)
\end{aligned}
$$




$$
\ll t^{-2 r \vartheta-2 r(1-\alpha)},
$$

provided $r \alpha>\frac{n}{2}$, so that (18) is convergent.

Relative to $S_{2}(\nu)$ we note that since we assume $t, t_{j}>0$, the second sum is empty and the first one is the sum over the $t_{j}$ such that $t-t^{\vartheta}<t_{j}<$ $t+t^{\vartheta}$, or equivalently, $a_{t, \vartheta}^{-}<\lambda\left(i t_{j}\right)<a_{t, \vartheta}^{+}$, where $a_{t, \vartheta}^{ \pm}=\left(t \pm t^{\vartheta}\right)^{2}+\rho^{2}$ and $\lambda\left(i t_{j}\right)=t_{j}^{2}+\rho^{2}$. Similarly, the sum of the integrals equals the integral taken over the set: $a_{t, \vartheta}^{-}<\lambda(i \mu)<a_{t, \vartheta}^{+}$. We note that, if $\mu$ is so that $\lambda(i \mu)$ is in this interval, we have, uniformly for $\nu \in S_{\sigma}$ (since $\sigma>0$ ),

$$
\left(s^{2}+(\mu-t)^{2}\right)^{r}\left(s^{2}+(\mu+t)^{2}\right)^{r}>D\left(1+t^{2 r}\right)
$$

with $D=D_{\sigma}$. We can use this estimate also in the terms of the sum, with $t_{j}$ in place of $\mu$. Therefore,

$$
\begin{aligned}
S_{2}(\nu) \ll(1+t)^{-2 r}\left(\sum_{a_{t, \vartheta}^{-}<\lambda\left(i t_{j}\right)<a_{t, \vartheta}^{+}}\left|\varphi_{j}(x)\right|^{2}\right. \\
\left.\quad+\sum_{\ell} c_{Q_{\ell}} \int_{a_{t, \vartheta}^{-}<\lambda(i \mu)<a_{t, \vartheta}^{+}}\left|E\left(Q_{\ell}, i \mu, x\right)\right|^{2} d \mu\right) \\
=(1+t)^{-2 r}\left(e\left(x, x, a_{t, \vartheta}^{+}\right)-e\left(x, x, a_{t, \vartheta}^{-}\right)\right) \\
\ll t^{-2 r}\left(\left(a_{t, \vartheta}^{+}-a_{t, \vartheta}^{-}\right)\left(t^{2}+\rho^{2}\right)^{n / 2-1}+\left(t^{2}+\rho^{2}\right)^{(n-1) / 2}\right) \\
\ll t^{n-1+\vartheta-2 r},
\end{aligned}
$$

for $\nu \in S_{\sigma}$, by (11).

The contribution $S_{e}(\nu)$ of the exceptional spectrum is $O\left(t^{-4 r}\right)$.

We have $S(\nu) \ll t^{-4 r}+t^{-2 \vartheta r-2 r+2 r \alpha}+t^{n-1+\vartheta-2 r}$, with the conditions $r>\frac{n}{4}, r \alpha>\frac{n}{2}, 0<\alpha<1$, and $0<\vartheta<1$. For a given small $\varepsilon>0$ and a given $r>\frac{n}{4}$, we first take $\alpha=\frac{1}{r}\left(\frac{n}{2}+\varepsilon\right)$. This imposes the condition $r>\frac{n}{2}+\varepsilon$ on $r$. Next, we take $\vartheta=\frac{2 \varepsilon+1}{2 r+1}$ so that the second and third summands be equal. If $\varepsilon$ is small enough, we have $0<\vartheta<1$. We end up with

$$
s(\nu) \tilde{\mathbf{P}}_{\nu}(x, y) \ll(1+|\nu|)^{\frac{n-1}{2}+\frac{\varepsilon+1 / 2}{2 r+1}} .
$$

We can take $r \in \mathrm{N}$ as large as we want, and obtain the estimate in the theorem.

\section{Distribution of Lattice Points.}

This section will be devoted to the proof of our main result.

We introduce the Dirichlet series

$$
\text { (20) } L(\nu)=L(x, y, \nu)=\sum_{\gamma \in \Gamma} a\left(x^{-1} \gamma y\right)^{-(\nu+\rho)}=\sum_{\gamma \in \Gamma} e^{-d(x K, \gamma y K)(\nu+\rho)}
$$


by (2). As shown in [MW], $\S 5$, this series converges absolutely, uniformly for $\operatorname{Re} \nu \geq \sigma+\rho$. The convergence is even uniform for $x$ and $y$ in compact sets. The series defines a holomorphic function in the half-plane $\{\nu: \operatorname{Re} \nu>\rho\}$. Moreover, $L(\nu)$ admits a meromorphic continuation to $\mathrm{C}$ and its poles in the open right half-plane are simple and located at $\nu_{j}-2 l>0$ where $l \in$ $\mathrm{N} \cup\{0\}, 0<\nu_{m} \quad \nu_{m-1} \cdots \quad \nu_{1}<\nu_{0}=\rho$ and $\lambda\left(\nu_{j}\right)$ is an exceptional eigenvalue of $C, 1 \quad j \quad m$. The desired result on the counting function $N(x, y ; r)$ will follow from some uniform estimates for $L(\nu)$ on vertical strips and a Tauberian theorem.

Theorem 4.1. Let $x, y \in X$, and let $N(x, y ; r)$ be as in the introduction. Let $\varphi_{j}, 1 \quad j \quad m$, be an orthonormal system of eigenfunctions of the Casimir operator $C$ with exceptional eigenvalues $\lambda\left(\nu_{j}\right)=\rho^{2}-\nu_{j}^{2}, 0<\nu_{j} \quad \rho$. Then

$$
\begin{aligned}
& N(x, y ; r)=\frac{\zeta 2^{1-n}}{2 \rho \operatorname{vol}(\Gamma \backslash X)} e^{2 \rho r} \\
& +\zeta 2^{1-n} \sum_{j=1}^{m} \frac{c\left(\nu_{j}\right)}{\nu_{j}+\rho} \varphi_{j}(x) \varphi_{j}(y) e^{\left(\rho+\nu_{j}\right) r}+O\left(e^{\left(2 \rho \frac{n}{n+1}+\varepsilon\right) r}\right)
\end{aligned}
$$

as $r \rightarrow \infty$, for any $\varepsilon>0$. Here $c(\nu)$ is the Harish-Chandra c-function, $n=\operatorname{dim} X$, and $\zeta=\operatorname{vol}(K / M)$ (see Section 2).

Proof. Fix - a compact subset of $G$ containing $x, y$ and let $\sigma>0$. Let $T$ be as in Part (iii) of Proposition 3.1. We note that

$$
\Gamma_{-}=\{\gamma \in \Gamma: d(u, \gamma v) \quad T+1, \text { for some } u, v \in-\}
$$

is a finite set. Since $\beta\left(x^{-1} \gamma y\right)=1$ for $\gamma \notin \Gamma_{\text {- }}$, we may write $L(\nu)$ for $\operatorname{Re} \nu>\rho$, as follows:

$$
\begin{array}{r}
L(\nu)=\sum_{\gamma \in \Gamma_{-}} a\left(x^{-1} \gamma y\right)^{-(\nu+\rho)}+\sum_{\gamma \in \Gamma-\Gamma_{-}}\left(a\left(x^{-1} \gamma y\right)^{-(\nu+\rho)}-Q_{\nu}\left(x^{-1} \gamma y\right)\right) \\
+s(\nu) \widetilde{\mathbf{P}}_{\nu}(x, y)-\sum_{\gamma \in \Gamma_{-}} \beta\left(x^{-1} \gamma y\right) Q_{\nu}\left(x^{-1} \gamma y\right)
\end{array}
$$

The first and fourth summands are given by finite sums and define holomorphic functions in the closed right half-plane, uniformly bounded on vertical strips. On the other hand, by using the expansion (3) for $Q_{\nu}(a)$ and the fact that the values $Q_{\nu}(g)$ are uniformly bounded on vertical strips (c.f. $[\mathrm{MW}])$ we see that the second term is estimated in absolute value by

$$
O\left(\sum_{\gamma \in \Gamma-\Gamma_{-}} a\left(x^{-1} \gamma y\right)^{-(\operatorname{Re} \nu+\rho+2-\varepsilon)}\right)
$$

for any positive $\varepsilon$, hence it is a holomorphic function in the region $\{\nu$ : $\operatorname{Re} \nu>\rho-2\}$, uniformly bounded on vertical strips with $\operatorname{Re} \nu \geq \rho-2+\varepsilon$ for each $\varepsilon>0$. Now, since $s(\nu) \widetilde{\mathbf{P}}_{\nu}(x, y)$ satisfies the estimate in Theorem 3.6, it follows from $(22)$ that $L(\nu)$ satisfies this same estimate, uniformly if $\rho-$ 
$2+\sigma \quad \operatorname{Re} \nu \quad \rho+\sigma$, for any positive $\sigma$. By an iteration of this argument, in which we treat leading terms in (3) separately, we conclude that there exists $C_{\sigma, \varepsilon}>0$ such that

$$
|L(x, y, \nu)| \quad \mathrm{C}_{\sigma, \varepsilon}|\operatorname{Im} \nu|^{\frac{n-1}{2}+\varepsilon}
$$

for $\operatorname{Im} \nu \geq 1, \sigma \quad \operatorname{Re} \nu \quad \rho+\sigma$. Furthermore the poles of $L(\nu)$ with $\operatorname{Re} \nu>$ $\rho-2$ coincide with those of $s(\nu) \widetilde{\mathbf{P}}_{\nu}(x, y)$ and lie at the $\nu_{j}, 0 j \quad j$, such that $\lambda\left(\nu_{j}\right)$ is an exceptional eigenvalue of $C$. (We here include $0=\lambda\left(\nu_{0}\right)$, with $\nu_{0}=\rho$, among the exceptional eigenvalues.) Also, the residue at such a pole $\mu$ is given by $\sum_{j, \nu_{j}=\mu} \zeta 2^{-p-q} c\left(\nu_{j}\right) \varphi_{j}(x) \varphi_{j}(y)$, by Proposition 3.3. In the case $j=0$, we have $\left|\varphi_{0}\right|=\operatorname{vol}(\Gamma \backslash X)^{-1 / 2}$, and residue $\zeta 2^{-p-q} / \operatorname{vol}(\Gamma \backslash X)$.

We now consider the Assumptions i), $\ldots$,iv) in the Appendix, in the case of $D(s)=L(s-\rho)$. So the region of absolute convergence is $\operatorname{Re} s>\tau:=2 \rho$.

As the set $\mathcal{C}$, we take $\left\{a\left(x^{-1} \gamma y\right)^{\alpha}: \gamma \in \Gamma\right\}$, and $a(c):=\#\{\gamma \in \Gamma$ : $\left.a\left(x^{-1} \gamma y\right)^{\alpha}=c\right\}$. The discreteness in Assumption i) follows from the fact that $\{\gamma \in \Gamma: d(x, \gamma y) \quad L\}$ is finite for each $L$.

The number $\tilde{\tau}$ in Assumption iii) can be any number $\tilde{\tau} \in(\rho, 2 \rho)$ not equal to some $\nu_{j}+\rho-2 \ell, \ell \geq 0$ integral.

The exponent $k$ in the estimate in Assumption iv) can be any $k>$ $\frac{n-1}{2}\left(2-\frac{\tilde{\tau}}{\rho}\right)$. We have used the Phragmen-Lindelöf theorem (see for instance [La], Ch.IX, §4, p.236.)

As the numbers $a(c)$ are positive, Proposition 5.1 in the Appendix yields

$$
\#\left\{\gamma \in \Gamma: a\left(x^{-1} \gamma y\right)^{\alpha} \quad X\right\}=\sum_{i} \frac{w_{i}}{s_{i}} X^{s_{i}}+O\left(X^{b}\right) \quad(X \rightarrow \infty),
$$

where $b=\frac{1}{k+1}(2 k \rho+\tilde{\tau}), s_{i}$ runs through the poles of $D(s)$ and $w_{i}$ denotes the residue at $s_{i}$. The optimal choice of $\tilde{\tau}$ is slightly larger than $\rho$. Thus we find

$$
\begin{aligned}
& \#\left\{\gamma \in \Gamma: a\left(x^{-1} \gamma y\right)^{\alpha} \quad X\right\} \\
& =\sum_{i} \frac{w_{i}}{s_{i}} X^{s_{i}}+O\left(X^{2 \rho-2 \rho /(n+1)+\varepsilon}\right) \quad(X \rightarrow \infty),
\end{aligned}
$$

for each $\varepsilon>0$. Only the $s_{i} \in\left(\frac{2 n}{n+1} \rho, 2 \rho\right]$ are relevant. The largest exponent is $s_{1}=\nu_{0}+\rho=2 \rho$.

For all rank-one groups, we have $2 \rho /(1+n)<2$. Indeed (see $[\mathrm{BM}]$, Table 1, p.110), if $G=S O_{F}(l, 1)$, with $F=\mathrm{R}, F=\mathrm{C}$ or $F=\mathrm{H}$, then $X \simeq F H^{l}$, we have $n=d l$ and $2 \rho=n-2+d, d=1,2,4$ respectively. If $G \simeq F_{4}^{1}$, then $2 \rho=22$ and $n=16$. This implies that all relevant terms in (24) correspond to eigenvalues of the Casimir operator: the set $\left\{s_{1}, \ldots, s_{q}\right\}$ (without multiplicities) is equal to the set $\left\{2 \rho, \nu_{1}+\rho, \ldots, \nu_{m}+\rho\right\}$ and furthermore, $w_{i}=\sum_{j, \nu_{j}+\rho=s_{i}} \zeta 2^{-p-q} c\left(\nu_{j}\right) \varphi_{j}(x) \varphi_{j}(y)$; in particular, $w_{0}=\frac{\zeta 2^{-p-q}}{\operatorname{vol}(\Gamma \backslash X)}$. 
To make the transition to $N(r)$, we take $X=e^{r}$ in the resulting asymptotic expression, and use that $a\left(x^{-1} \gamma y\right)^{\alpha}=e^{d(x K, \gamma y K)}$. The result is

$$
\begin{aligned}
\#\{\gamma & \in \Gamma: d(x K, \gamma y K) \quad r\}=\frac{\zeta 2^{-p-q}}{2 \rho \operatorname{vol}(\Gamma \backslash X)} e^{2 \rho r} \\
& +\sum_{j=1}^{m} \frac{\zeta 2^{-p-q} c\left(\nu_{j}\right) \varphi_{j}(x) \varphi_{j}(y)}{\nu_{j}+\rho} e^{\left(\nu_{j}+\rho\right) r}+O\left(e^{r\left(2 \rho \frac{n}{n+1}+\varepsilon\right)}\right),
\end{aligned}
$$

as $r \rightarrow \infty$, for any $\varepsilon>0$. The theorem is now proved.

Remarks 4.2. (i) We compare Theorem 4.1 with [LP], (1.3) in the case when $G=S O(n, 1)$. The theorem of Lax-Phillips implies that

$$
\begin{gathered}
N(r)=\sum_{j=0}^{m} \frac{\zeta \Gamma\left(\rho+\frac{1}{2}\right) \Gamma\left(\nu_{j}\right)}{2 \sqrt{\pi} \Gamma\left(\nu_{j}+\rho+1\right)} \varphi_{j}(x) \varphi_{j}(y) e^{\left(\nu_{j}+\rho\right) r} \\
+O\left(r^{\frac{3}{n+1}} e^{r\left(2 \rho \frac{n}{n+1}\right)}\right) \quad(r \rightarrow \infty) .
\end{gathered}
$$

In this case, $2 \rho=p=n-1$ and $c(\nu)=\frac{\Gamma\left(\rho+\frac{1}{2}\right) \Gamma(\nu)}{2^{-p} 2 \sqrt{\pi} \Gamma(\nu+\rho)}$ (see [He2], Thm 6.14 ; recall that $c(\rho)=1)$. As all roots have the same length, $\zeta=\operatorname{vol}(K / M)$ is equal to the volume of the unit sphere in $p$ with respect to the measure induced by restriction of the form $B$ to $p$. Thus,

$$
\frac{\zeta 2^{-p-q} c\left(\nu_{j}\right)}{\nu_{j}+\rho}=\frac{\zeta \Gamma\left(\rho+\frac{1}{2}\right) \Gamma\left(\nu_{j}\right)}{2 \sqrt{\pi} \Gamma\left(\nu_{j}+\rho+1\right)}
$$

hence Theorem 4.1 yields (26) with $e^{\varepsilon r}$, for any $\varepsilon>0$, in place of $r^{\frac{3}{n+1}}$.

(ii) The determination of the optimal estimate for the error term in (21) is an open problem. In the case when $\Gamma$ is cocompact, this question was studied by Huber ([Hbr]) for $X=H^{2}$, by Margulis ([Ma]) for manifolds of negative curvature), by Günther (for $X$ as above) and by Bartels ([B]) when $X$ is a general symmetric space of non positive curvature. In the case when $G=S O(n, 1)$ and $n \geq 3$, the best result is (26). In the case when $n=2$, Selberg (unpublished) had before obtained the same exponential term $e^{\frac{2}{3} r}$ times a lower power of $r$ (see[LP], Introduction). We should also remark that Lax and Phillips work with the condition that $\Gamma$ be geometrically finite, which is weaker than finite volume. We need the finite volume condition to be able to use the spectral theory of $L^{2}(\Gamma \backslash X)$ and the results in [MW]. However, many of the arguments work in a greater generality. By refining the methods in this paper, one should be able to extend Theorem 4.1 to any $\Gamma$ satisfying the Lax-Phillips condition.

(iii) The formulation of Theorem 4.1 depends on our normalization of the Riemannian structure. Let us consider what happens if we replace the distance by $d^{\tau}(x, y)=\tau d(x, y)$ for some $\tau>0$, that is, if we let the new metric be $B^{\tau}=\tau^{2} B$. 
The Riemannian measure changes according to $d^{\tau} x=\tau^{n} d x$. So the factors $\frac{1}{\operatorname{vol}(\Gamma \backslash X)}$ and $\varphi_{j}(x) \varphi_{j}(y)$ in $(21)$ are multiplied by $\tau^{-n}$.

We have to replace $\zeta$ by $\zeta^{\tau}=\operatorname{vol}^{\tau}(K / M)$, where the measure is induced by the restriction of $-B^{\tau}$ to $\mathrm{k} \cap \mathrm{m}^{\perp}$. Hence $\zeta^{\tau}=\tau^{\operatorname{dim}(\mathrm{k} / \mathrm{m})} \zeta=\tau^{n-1} \zeta$.

The Casimir operator associated to $B^{\tau}$ is $C^{\tau}=\tau^{-2} C$. So the eigenvalues are multiplied by $\tau^{-2}$, and the spectral parameter satisfies $\nu^{\tau}=\tau^{-1} \nu$. In the new norm, the length of the simple root $\alpha$ is equal to $\tau^{-1}$, and the identification between $\mathrm{C}$ and $\mathrm{a}_{c}^{*}$ is given by $\nu^{\tau} \mapsto \nu^{\tau} \tau \alpha$. The Harish Chandra function is a function on $\mathrm{a}_{c}^{*}$, so we have $c\left(\nu^{\tau} \tau \alpha\right)=c(\nu \alpha)$.

The factors $\frac{1}{2 \rho}$ and $\frac{1}{\nu_{j}+\rho}$ in $(21)$ are multiplied by $\tau$, which makes that the coefficients $c_{0}, c_{1}, \ldots$ in the asymptotic expansion do not change under the normalization.

The counting function becomes $N^{\tau}(x, y, r)=N(x, y, r / \tau)$. Inserting $\frac{r}{\tau}$ for $r$ into the exponential factors in (21), changes these into respectively $e^{2 \rho^{\tau} r}, e^{\left(\rho^{\tau}+\nu_{j}^{\tau}\right) r}$ and $e^{\left(2 \rho^{\tau} \frac{n}{n+1}+\frac{\varepsilon}{\tau}\right) r}$. So Theorem 4.1 does not depend on the choice of the Riemannian metric.

\section{Appendix. A Tauberian Result.}

The purpose of this section is to study the asymptotic properties of the partial sums of the coefficients of a Dirichlet series satisfying i),...,v) below. We make no claim to originality, but we include a proof, since we have been unable to find a reference for the result we need.

We start with the following assumptions:

i) $\mathcal{C} \subset(0, \infty)$ is a closed infinite discrete set in $R$. So $\infty$ is its only accumulation point.

ii) Let $a: \mathcal{C} \rightarrow[0, \infty)$. The Dirichlet series $D(s):=\sum_{c \in \mathcal{C}} a(c) c^{-s}$ converges absolutely on the region $\operatorname{Re} s>\tau$, with $\tau>0$.

iii) $D$ has a meromorphic continuation to the half plane $\operatorname{Re} s \geq \tilde{\tau}$, with $0<\tilde{\tau}<\tau$. In this half plane, there are finitely many first order poles at points $s_{1}=\tau, s_{2}, \ldots, s_{q}$, with $\operatorname{Re} s_{m} \in(\tilde{\tau}, \tau]$. Let $w_{m}$ be the residue at $s_{m}$.

iv) $D(s)=O_{\sigma}\left((1+|\operatorname{Im} s|)^{k}\right)$ on a strip $\tilde{\tau} \quad \operatorname{Re} s \quad \sigma$, outside a neighborhood of the singularities, with $\sigma>\tau$.

Proposition 5.1. If the conditions i)-iv) are satisfied, then

$$
\sum_{c \quad X} a(c)=\sum_{m=1}^{q} \frac{w_{m}}{s_{m}} X^{s_{m}}+O\left(X^{b}\right) \quad(X \rightarrow \infty) .
$$

with $b=\frac{k \tau+\tilde{\tau}}{k+1}$

Only the $m$ with $\operatorname{Re} s_{m}>b$ are relevant.

The condition $a(c) \geq 0$ in Assumption ii) is necessary. We shall start the proof with arbitrary $a(c) \in \mathrm{C}$ such that the series converges absolutely. This also means that at first the maximum of $\operatorname{Re} s_{m}$ need not be equal to $\tau$. 
Proof. The main idea is to approximate $A(X):=\sum_{c}{ }_{X} a(c)$ by

$$
S(\psi):=\sum_{c \in \mathcal{C}} a(c) \psi(c),
$$

for suitable $\psi \in C_{c}^{\infty}(0, \infty)$.

For any such $\psi$, the Mellin transform

$$
\mathcal{M} \psi(s):=\int_{0}^{\infty} x^{s} \psi(x) \frac{d x}{x}
$$

is quickly decreasing on vertical strips. The inversion is given by

$$
\psi(x)=\frac{1}{2 \pi i} \int_{\operatorname{Re} s=\sigma} x^{-s} \mathcal{M} \psi(s) d s
$$

for any $\sigma \in \mathrm{R}$. This implies $S(\psi)=\frac{1}{2 \pi i} \int_{\operatorname{Re} s=\sigma} D(s) \mathcal{M} \psi(s) d s$ for each $\sigma>\tau$. Assumption iv) shows that we can transform this into

$$
S(\psi)=\sum_{m=1}^{q} w_{m} \mathcal{M}\left(s_{m}\right)+\frac{1}{2 \pi i} \int_{\operatorname{Re} s=\tilde{\tau}} D(s) \mathcal{M} \psi(s) d s .
$$

The following choice of the test function $\psi$ has examples in the work of Iwaniec. $\psi=\psi_{X, Y}$ depends on two large parameters $X \geq 10, Y \geq 10$. It is an element of $C_{c}^{\infty}(0, \infty)$ approximating the characteristic function of the interval $[0, X]$. The auxiliary parameter $Y$ governs the steepness on the right. Put $\mu:=\min (\mathcal{C})$. The properties of $\psi=\psi_{X, Y}$ are

a) $0 \quad \psi, \psi=0$ on $[0, \mu / 2]$ and on $[X+X / Y, \infty)$, and $\psi=1$ on $[\mu, X]$.

b) On $[0, \mu]$ the function $\psi_{X, Y}$ does not depend on $X$ and $Y$.

c) On $[X, \infty)$ we take $\psi_{X, Y}(X+x):=\omega(Y x / X)$, where $\omega \in C^{\infty}(0, \infty)$ is a decreasing function, equal to 1 on a neighborhood of 0 , and equal to 0 on $[1, \infty)$.

This implies that $\left|\psi^{(\ell)}\right| \ll Y^{\ell} X^{-\ell}$ on $[X, X+X / Y]$.

We derive some estimates for the Mellin transform of this test function. For all $t \in \mathrm{R}$ we have

$$
|\mathcal{M} \psi(\tilde{\tau}+i t)| \quad \int_{0}^{X+X / Y} x^{\tilde{\tau}} \frac{d x}{x} \ll_{\tilde{\tau}} X^{\tilde{\tau}} .
$$

For $\ell \geq 1$, and $\sigma>0$ we have

$$
\begin{gathered}
\left|\mathcal{M} \psi^{(\ell)}(\sigma+i t)\right| \quad\left(\int_{0}^{\mu}+\int_{X}^{X+X / Y}\right) x^{\sigma}\left|\psi^{(\ell)}(x)\right| \frac{d x}{x} \\
\ll_{\sigma, \ell} 1+Y^{\ell} X^{-\ell} X^{\sigma} Y^{-1} \ll 1+Y^{\ell-1} X^{\sigma-\ell} .
\end{gathered}
$$

From $\mathcal{M} \psi^{(\ell)}(s)=(s-1) \cdots(s-\ell) \mathcal{M} \psi(s-\ell)$ we conclude that

$$
|\mathcal{M} \psi(\tilde{\tau}+i t)| \ll_{\tilde{\tau}, \ell} Y^{\ell-1} X^{\tilde{\tau}}(1+|t|)^{-\ell}
$$

for each $\ell \geq 1$. 
We estimate the integral

$$
\frac{1}{2 \pi i} \int_{\operatorname{Re} s=\tilde{\tau}} D(s) \mathcal{M} \psi(s) d s
$$

by using various bounds in the cases $|\operatorname{Im} s| \in[0,1],[1, Y]$, and $[Y, \infty)$. From (32) we obtain for the integral over $[0,1]$ the bound $X^{\tilde{\tau}}$. For $[1, Y]$ we use (33) with $\ell=1$ and find $\int_{1}^{Y} X^{\tilde{\tau}} t^{k-1} d t \ll X^{\tilde{\tau}} Y^{k}$. For the last interval we use $\ell=L+1$ with $L \in(k, k+1]$. This gives $\int_{Y}^{\infty} Y^{L} X^{\tilde{\tau}} t^{k-L-1} d t \ll Y^{k} X^{\tilde{\tau}}$. Thus we obtain

$$
\frac{1}{2 \pi i} \int_{\operatorname{Re} s=\tilde{\tau}} D(s) \mathcal{M} \psi(s) d s \ll X^{\tilde{\tau}} Y^{k} .
$$

For $\operatorname{Re} s=\sigma \in(\tilde{\tau}, \tau]$, we have the following estimate:

$$
\begin{aligned}
\mathcal{M} \psi(s) & =\int_{0}^{X} x^{s-1} d x+O(1)+O\left(\int_{X}^{X+X / Y} x^{\sigma-1} d x\right) \\
& =\frac{X^{s}}{s}+O\left(1+X^{\sigma} Y^{-1}\right) .
\end{aligned}
$$

We apply this to the terms $w_{m} \mathcal{M}\left(s_{m}\right)$ in (31), and obtain the final estimate of $S(\psi)$ :

$$
S\left(\psi_{X, Y}\right)=\sum_{m=1}^{q} \frac{w_{m}}{s_{m}} X^{s_{m}}+O\left(X^{\tilde{\tau}} Y^{k}+X^{\eta} Y^{-1}\right),
$$

where $\eta:=\max _{1} m_{q} \operatorname{Re} s_{m}$. If there are no singularities, then $S(\psi)=$ $O\left(X^{\tilde{\tau}} Y^{k}\right)$.

The problem left is to estimate the difference between $S\left(\psi_{X, Y}\right)$ and $A(X)$. Without the condition $a(c) \geq 0$, we do not see another way than to estimate the difference trivially:

$$
\begin{aligned}
& |A(X)-S(\psi)| \sum_{X<c}|a(c)| \\
& \ll X^{\sigma} \sum_{X<c}|a(c)| c^{-\sigma}=X^{\sigma} \cdot o(1),
\end{aligned}
$$

for any $\sigma>\tau$. As we do not have precise information on $o(1)$, even the explicit terms in (35) may be overwhelmed by the difference $A(X)-S(\psi)$.

Now we use the non-negativity in Assumption ii). The right-most $s_{m}$, say $s_{1}$, coincides with $\tau$. Hence $\eta=\tau$ in (35). We have $S\left(\psi_{Z, Y}\right) \quad A(X)$ $S\left(\psi_{X, Y}\right)$, with $Z=X /(1+1 / Y)$. We find

$$
\begin{aligned}
S & \left(\psi_{X, Y}\right)-S\left(\psi_{Z, Y}\right) \\
& =\sum_{m=1}^{q} \frac{w_{m}}{s_{m}} X^{s_{m}}\left(1-\left(1+\frac{1}{Y}\right)^{-s_{m}}\right)+O\left(X^{\tilde{\tau}} Y^{k}+X^{\tau} Y^{-1}\right) \\
& =O\left(X^{\tilde{\tau}} Y^{k}+X^{\tau} Y^{-1}\right) .
\end{aligned}
$$


So the estimate of $S\left(\psi_{X, Y}\right)$ in (35) is valid for $A(X)$ as well. The choice $Y=X^{(\tau-\tilde{\tau}) /(k+1)}$ gives the assertion in the proposition.

Integrality assumption. In the application of the present paper, it is improbable that the elements of $\mathcal{C}$ are integral. But in an arithmetic situation, one may obtain better estimates than in (36), without the assumption $a(c) \geq 0$. We need information on the spacing of the $c \in \mathcal{C}$, and on the growth of the $a(c)$. We give the following result as an example.

Proposition 5.2. Suppose that $\mathcal{C}=\mathrm{N}$ in Assumption i), and that $a(n) \in \mathrm{C}$, $a(n)=O\left(n^{\tau-1}\right)$, in Assumption ii). With these modifications, Assumptions i) -iv) imply

$$
\sum_{n<X} a(n)=\sum_{m=1}^{q} \frac{w_{m}}{s_{m}} X^{s_{m}}+O\left(X^{b_{1}}\right) \quad(X \rightarrow \infty),
$$

with $b_{1}=\max ((k \tau+\tilde{\tau}) /(k+1), \tau-1)$.

Proof. We now have $\sum_{X<c \quad X+X / Y}|a(c)| \ll(1+X / Y)(X+X / Y)^{\tau-1}$, and obtain instead of (36):

$$
|A(X)-S(\psi)| \ll X^{\tau} Y^{-1}+X^{\tau-1} .
$$

We combine this with (35) to obtain the proposition.

\section{REFERENCES}

[B] Bartels H.-J., Nichteuklidische Gitterpunktprobleme und Gleichverteilung in linearen algebraischen gruppen, Comment. Math. Helvetici 57, 1982, 158-172.

[Bo] Borel A., Introduction aux groupes arithmétiques, Publ. Inst. Math. Strasbourg, 1969. [BM] Bruggeman R.W., Miatello R.J., Estimates of Kloosterman Sums for groups of Real Rank One, Duke Math. J. 80, 1995, 105-137.

[GR] Garland H., Raghunathan M.S., Fundamental domains for lattices in (R-)rank 1 semisimple Lie groups, Ann. of Math. 92 (1970) 279-326.

[G] Günther P., Gitterpunktprobleme in symmetrischen Riemannschen Räumen vom Rang 1, Math. Nachrichten 94, 5-27, 1980.

[He] Helgason S., Differential Geometry and Symmetric Spaces, Academic Press, San Diego, 1962.

[He2] Helgason S., Groups and Geometric Analysis, Academic Press, San Diego, 1984.

[Hbr] Huber H., Über eine neue Klasse automorpher Funktionen und ein Gitterpunktproblem in der hyperbolischen Ebene, I., Comment. Math. Helvetici 30, 1955, 20-62.

[Hö] Hörmander L., The spectral function of an elliptic operator, Acta Math. 121, 193-218 (1968).

[La] Lang S., Complex Analysis, Addison-Wesley, 1977.

[Langl] Langlands R.P., On the Functional Equations Satisfied by Eisenstein Series; Lect. Notes Math. 544, Springer-Verlag, 1976.

[LP] Lax P., Phillips R., The Asymptotic Distribution of Lattice Points in Euclidean and Non-Euclidean Spaces, Jour. Funct. Anal. 46, 280-350, 1982.

[Le] Levitan B., Asymptotic formulas for the number of lattice points in Euclidean and Lobachevskii spaces (Russian), Uspekhi Mat. Nauk 42, vol. 3(255) , 13-38, 1987; translation in Russ. Math. Surveys 42:3 (1987) 13-42. 
[Ma] Margulis G., Applications of ergodic theory to the investigation of manifolds of negative curvature (Russian), Funkt. Anal. i Ego Prilozh. 3, vol. 4 (1969) 89-90; translation in Funct. Anal. Applic. 3, 335-336, 1969.

[MW] Miatello R.J., Wallach N.R., The resolvent of the Laplacian on locally symmetric spaces, Jour. Differential Geometry 36, 663-698, 1992.

[Pa] Patterson S.J., A lattice-point problem in hyperbolic space, Mathematika 22, 81-88, 1975.

Mathematisch Institudt, Universiteit Utrecht, Postbus 80010, NL-3508 TA UTRECHT, NEDERLAND

E-mail address: bruggeman@math.uu.nl

Facultad de Matemática, Astronomía y Física, Universidad Nacional de Córdoba, Córdoba 5000, Argentina

E-mail address: miatello@mate.uncor.edu

Department of Mathematics, University of California San Diego, La Jolla, CALifornia 92093, U.S.A

E-mail address: nwallach@math.ucsd.edu 